\title{
A Therapeutic Microneedle Patch Made from Hair-Derived Keratin for Promoting Hair Regrowth
}

Guang Yang ${ }^{\dagger, \xi,+,}$, Qian Chen ${ }^{\dagger, \xi,}$, Di Wen ${ }^{\dagger, \xi, t}$, Zhaowei Chen ${ }^{\dagger, \xi,}$, Jinqiang Wang ${ }^{\dagger, \xi, t}$, Guojun

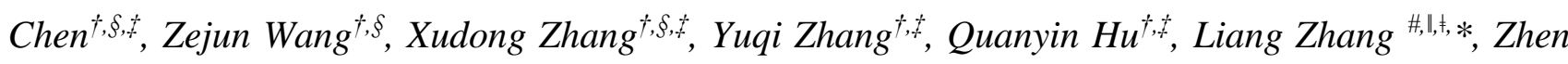
$G u^{\dagger, \xi, \dot{t}, *}$

${ }^{\dagger}$ Department of Bioengineering, University of California, Los Angeles, California 90095, United States

$\S$ California NanoSystems Institute, Jonsson Comprehensive Cancer Center and Center for Minimally Invasive Therapeutics, University of California, Los Angeles, California 90095, United States

\$ Joint Department of Biomedical Engineering, University of North Carolina at Chapel Hill and North Carolina State University, Raleigh, North Carolina 27695, United States

${ }^{\perp}$ College of Chemistry, Chemical Engineering and Biotechnology, Donghua University, Shanghai, 201620, People's Republic of China

\# CAS Key Laboratory of Tissue Microenvironment and Tumor, Shanghai Institute of Nutrition and Health, Shanghai Institutes for Biological Sciences, University of Chinese Academy of Sciences, Chinese Academy of Sciences, Shanghai, 200031, People's Republic of China

" Institute for Stem Cell and Regeneration, Chinese Academy of Sciences, 1 Beichen West Road, Chaoyang District, Beijing, 100101, People's Republic of China

${ }^{\ddagger}$ Shanghai Institutes for Biological Sciences - Changzheng Hospital Joint Center for Translational Research, Institutes for Translational Research (CAS-SMMU), Shanghai, 200433, People's Republic of China

*Corresponding author. E-mail: guzhen@ucla.edu (Z.G.) and Zhangliang01 @ sibs.ac.cn (L.Z.) 


\section{Experimental section}

\section{Preparation and characterization of UK5099-loaded PLGA nanoparticles}

UK5099-loaded PLGA nanoparticles were prepared via an emulsion/solvent evaporation method. Briefly, $5 \mathrm{mg}$ PLGA and $0.2 \mathrm{mg}$ UK5099 were dissolved in $0.4 \mathrm{~mL}$ dichloromethane, followed by addition of $1 \mathrm{~mL} 3 \%$ poly(vinyl alcohol) (PVA) solution. Subsequently, the mixture was sonicated and dispersed into $4 \mathrm{~mL} 0.3 \%$ PVA solution under stirring. Finally, the mixture was applied to the rotary evaporator for dichloromethane evaporation. The morphology and size of the resultant nanoparticles were characterized by transmission electron microscopy and dynamic light scattering analysis, respectively. The quantitative analysis of UK5099 was performed by high performance liquid chromatography (HPLC).

\section{Loading amount of exosomes and UK5099 in the microneedles}

The loading amount of cargoes in the microneedles was defined as the difference between the cargo loading in the whole MN patch and that in the patch base. The total amount of cargoes added in the preparation of the MN patch was considered as the cargo loading in the whole MN patch. For detection of the loading amount in the patch base, the MN patch loaded with Dil-exosomes and UK5099-loaded PLGA NPs was first inserted into the mouse skin for $4 \mathrm{~h}$, followed by removal of the patch base. Then, the patch base was immersed into PBS solution. The amounts of exosomes and UK5099 in the solution were analyzed by fluorescence and HPLC, respectively.

\section{In vitro release study}

The Dil labeled-exosomes were used in this test. For the in vitro release of Dil labeled-exosomes or UK5099, the needle tips of the MN patch were immersed in the PBS solution at $37{ }^{\circ} \mathrm{C}$. At a predetermined time point, the PBS solution was collected and the same volume of fresh PBS solution was added. The concentration of Dil labeled-exosomes and UK5099 in the PBS solution was determined by fluorescence and HPLC, respectively. The released percentage of Dil labeledexosomes or UK5099 was recorded at each timepoint, by taking the loading amount of Dil labeledexosomes or UK5099 in the microneedles as $100 \%$.

\section{In vivo release study}

The Dil labeled-exosomes were used in this test. For the MN administration, the patch loaded with Dil labeled-exosomes was inserted into the shaved mouse skin. The patch base was removed at $4 \mathrm{~h}$ postinsertion into the skin. Fluorescence imaging was monitored at Day 1, 4, 7 and 10 by an IVIS 
Spectrum imaging system (Perkin Elmer). The subcutaneous injection administration with the same amount of Dil labeled-exosomes was performed for comparison. Three mice were tested for each administration group. The relative fluorescence intensities of Dil labeled-exosomes in each group were recorded at each timepoint, by taking the fluorescence intensity of Dil labeled-exosomes at Day 1 via the HMN administration as $100 \%$.

\section{In vivo degradation study of the MN patch}

To investigate the in vivo degradation of $\mathrm{MN}$ patch including the HMN and PMN patch, the microneedles were fabricated by Cy5.5-labeled keratin. In this test, the MN patch was inserted into the shaved mouse skin. The patch base was removed at $4 \mathrm{~h}$ postinsertion into the skin. Fluorescence imaging was monitored at Day 1, 4, 7 and 10 by an IVIS Spectrum imaging system (Perkin Elmer). The relative fluorescence intensities of Cy5.5-labeled microneedles were recorded at each timepoint, by taking the fluorescence intensity of Cy5.5-labeled microneedles at Day 1 via the HMN administration as $100 \%$. Three mice were tested for each group.

\section{MTT assay}

The human dermal fibroblast cell was used as the model cell for MTT assay. The cell viability was determined according to our previous work. ${ }^{1}$ After grown to $70 \%$ confluence, the cells were incubated in sample solutions at $37{ }^{\circ} \mathrm{C}$ with $5 \% \mathrm{CO}_{2}$ for $48 \mathrm{~h}$, including PBS, soak solution of empty HMN needle tips in PBS, soak solution of UK5099-loaded HMN in PBS, soak solution of exosomesloaded HMN in PBS, and pure UK5099 or exosomes with the same dosage in HMN. The soak solution of the HMN system was obtained by immersing it in PBS for $50 \mathrm{~h}$.

\section{Western blot}

Mice skins were ground and lysed with protease and phosphatase inhibitors. Equal amounts of proteins were separated on SDS-polyacrylamide gel electrophoresis and transferred to PROTRAN nitrocellulose membrane. The membrane was blocked with $3 \%$ nonfat dry milk for $1 \mathrm{~h}$, and incubated overnight at $4{ }^{\circ} \mathrm{C}$ with primary antibodies targeting $\beta$-catenin, PCNA, K15, CD34, and ALP, respectively. Antibody to mouse $\beta$-actin was used as a control. All the antibodies were purchased from Santa Cruz Biotechnology and diluted at 1:500 in 1.5\% BSA solution. The membranes were washed three times and incubated with horseradish peroxidase-conjugated antimouse (1:2000; Seracare) IgG secondary antibodies for $1 \mathrm{~h}$ at room temperature.

\section{Histology and immunostaining}


For histopathology, the harvested skins were fixed in $10 \%$ formalin and paraffin-embedded, sectioned and stained with hematoxylin and eosin by NCSU CVM Histopathology Lab. Histopathology images were acquired on EVOS FL fluorescence microscopy (Invitrogen). For immunostaining, the harvested skins were embedded in OCT, frozen, and cryosectioned (15 $\mu \mathrm{m})$. All sections for staining were fixed in $4 \%$ paraformaldehyde for $10 \mathrm{~min}$, permeabilized in PBST (PBS + $0.3 \%$ Triton), and blocked in FBS for another $10 \mathrm{~min}$. Then, the sections were incubated overnight at $4{ }^{\circ} \mathrm{C}$ with primary antibodies targeting CD3 (Rat, 1:100; eBioscience) and CD68 (Rat, 1:100; Biolegend). After incubation, the sections were rinsed with PBST and incubated with 1:200 diluted Rhodamine-conjugated IgG secondary antibody at room temperature for $90 \mathrm{~min}$ and counterstained with DAPI for $5 \mathrm{~min}$. The fluorescent signals were visualized using EVOS FL fluorescence microscopy (Invitrogen).

\section{Reference}

(1) Yang, G.; Yao, Y.;Wang, X. Comparative Study of Kerateine and Keratose Based Composite Nanofibers for Biomedical Applications. Mater. Sci. Eng., C 2018, 83, 1-8. 


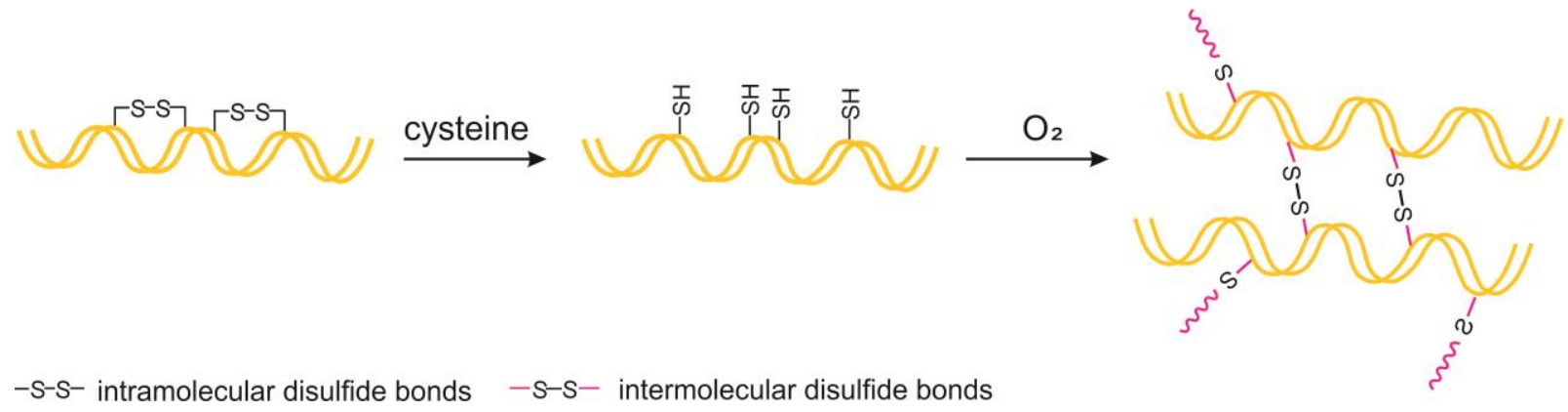

Figure S1. The schematic illustration of the formation mechanism of keratin hydrogel by 8 wt $\%$ keratin and $0.4 \mathrm{wt} \%$ cysteine. The inherent intramolecular disulfide bonds in keratin were cleaved by cysteine into free thiols, which could further form intermolecular disulfide bonds through the thiol oxidation.

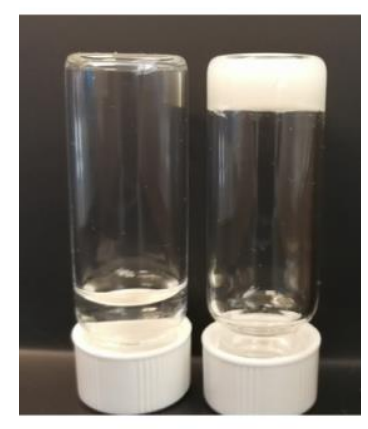

Figure S2. Photograph of keratin hydrogel prepared by 8 wt $\%$ keratin and 0.4 wt $\%$ cysteine (right). The same keratin concentration without cysteine cannot form hydrogel (left).

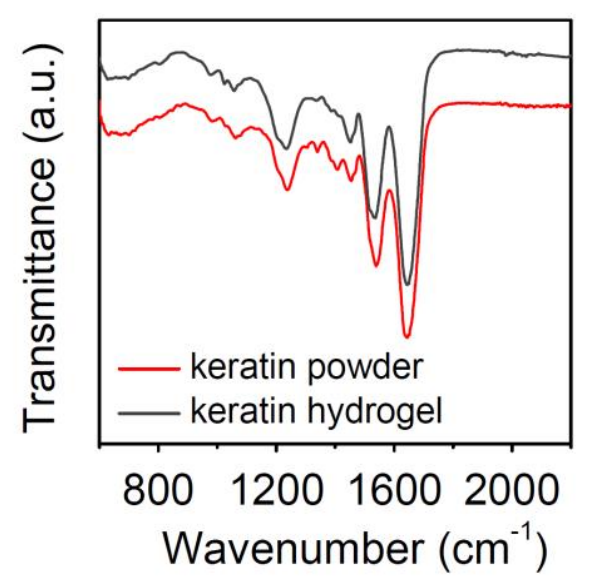

Figure S3. FTIR spectra of keratin hydrogel and keratin powder. 


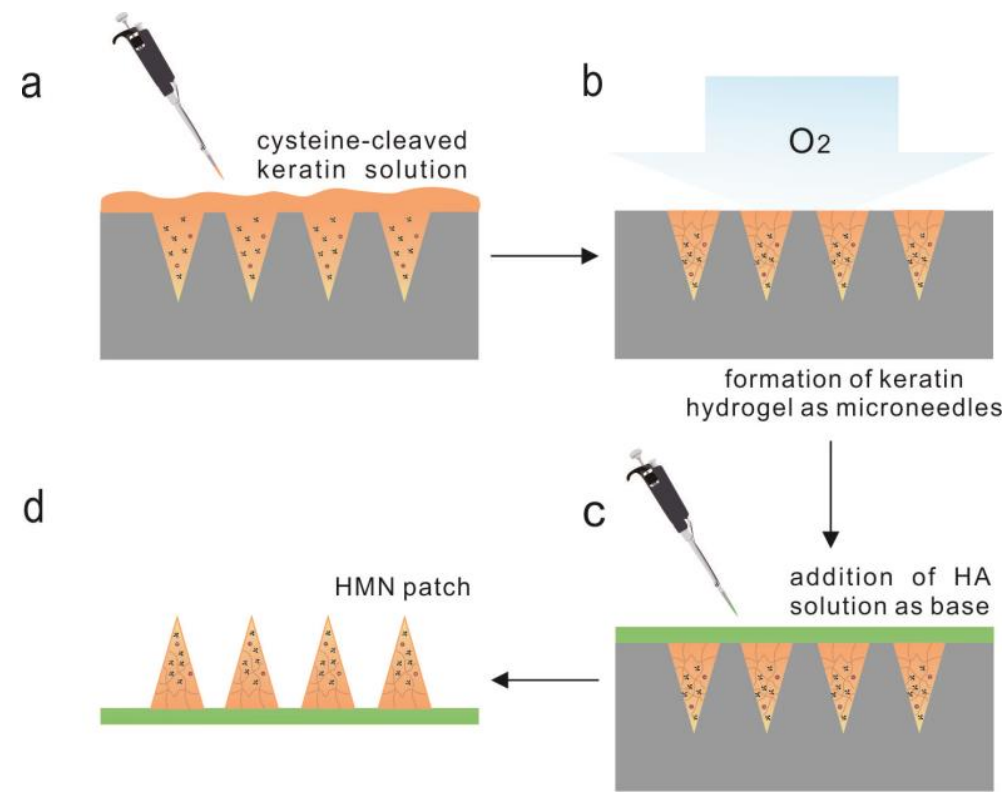

Figure S4. Preparation process of a detachable HMN patch. a: $50 \mu \mathrm{L}$ of keratin solution containing cysteine, exosomes and UK5099-loaded PLGA nanoparticles was deposited into the needle cavities. Then, another $50 \mu \mathrm{L}$ of keratin solution containing cysteine was deposited to fill the needle cavities, followed by removal of the excessive keratin solution outside the cavities via a plastic scraper. b: the keratin solution was kept in air for $1 \mathrm{~h}$ to form keratin hydrogel. c: $1 \mathrm{~mL}$ of hyaluronic acid (HA) solution was added onto the micromold and allowed to dry at room temperature. d: The MN patch was detached from the silicone mold after complete desiccation.

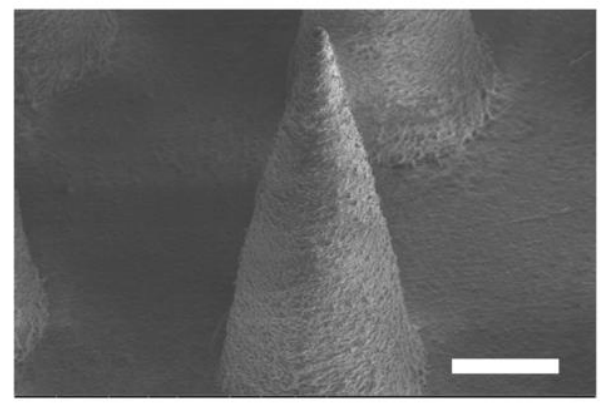

Figure S5. SEM image of the needle tip of the HMN patch. Scale bar: $250 \mu \mathrm{m}$. 

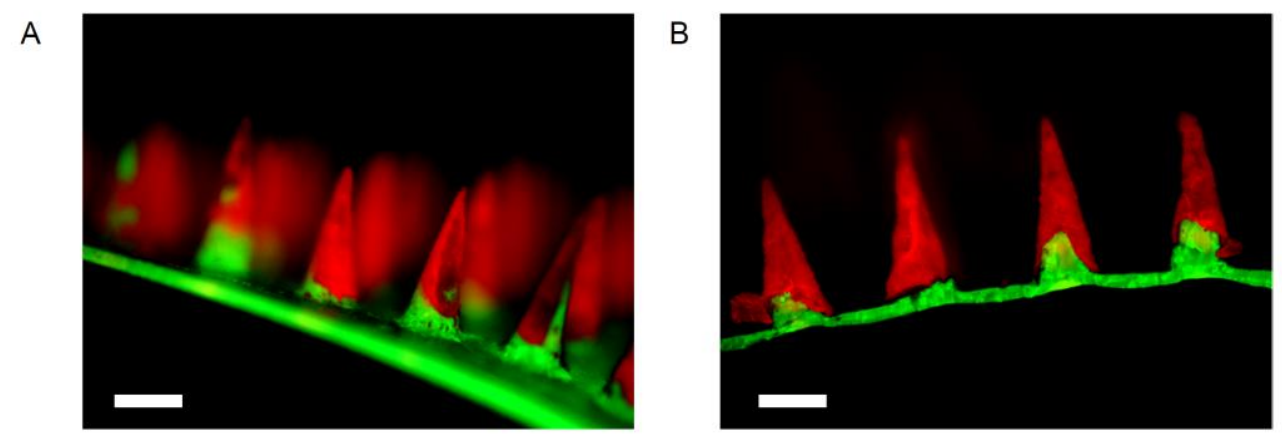

Figure S6. Fluorescence images of a representative PMN patch fabricated by rhodamine B-labeled keratin (microneedles) and FITC-labeled hyaluronic acid (patch base) from different angles. Scale bar: $250 \mu \mathrm{m}$.

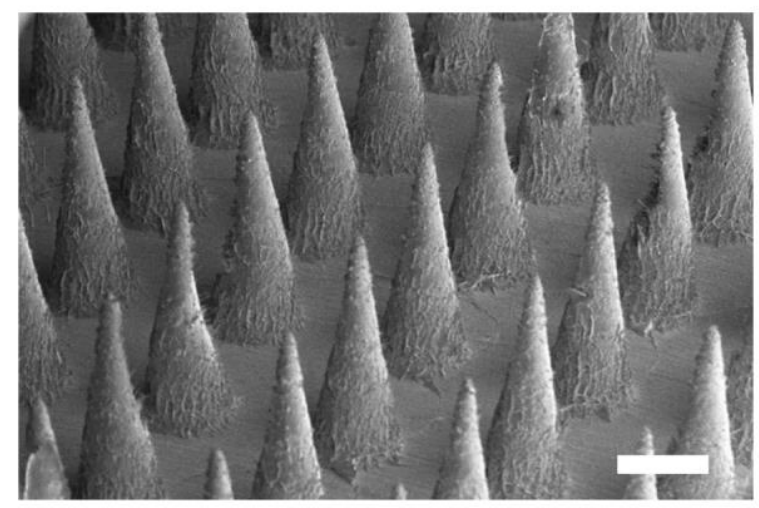

Figure S7. SEM image of the PMN patch. Scale bar: $250 \mu \mathrm{m}$.
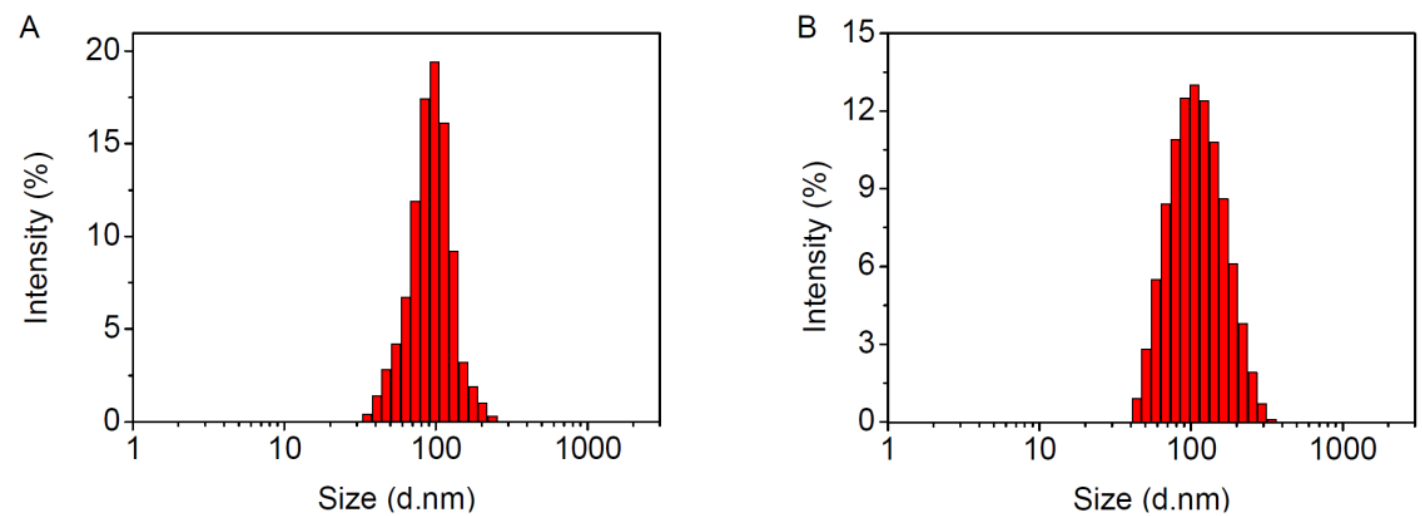

Figure S8. Particle size distribution of exosomes (A) and UK5099-loaded PLGA NPs (B) measured by the dynamic light scattering (DLS). 


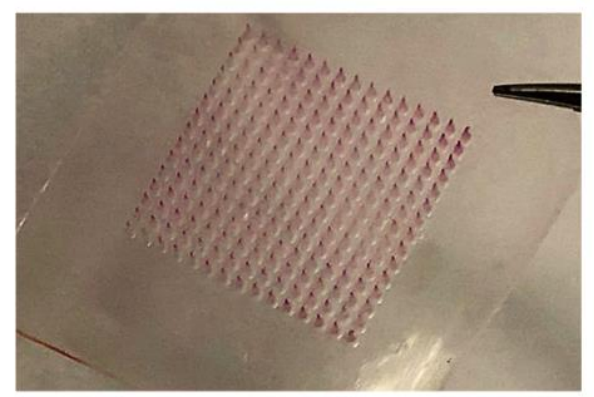

Figure S9. Photograph of a representative HMN patch loaded with Dil-exosomes and UK5099loaded PLGA NPs.
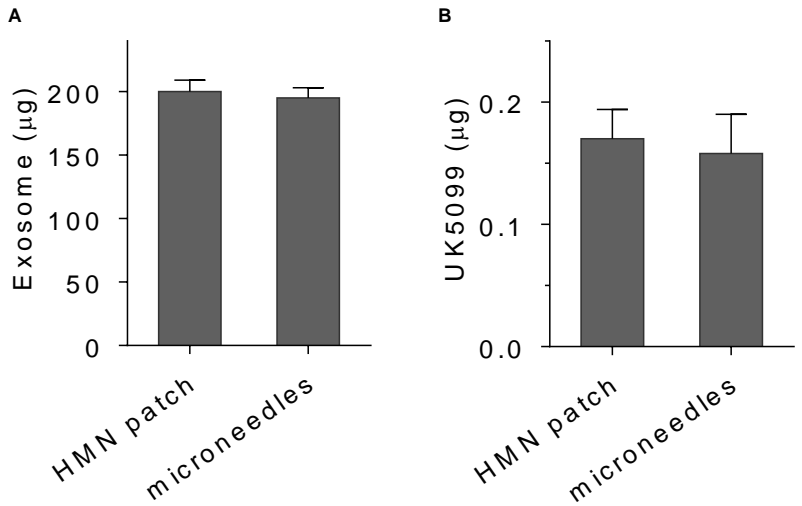

Figure S10. Loading capacities of exosomes (A) and UK5099 (B) in the whole HMN patch and microneedles, respectively. Data points represent mean $\pm \operatorname{SD}(n=3)$. Error bars indicate SD.

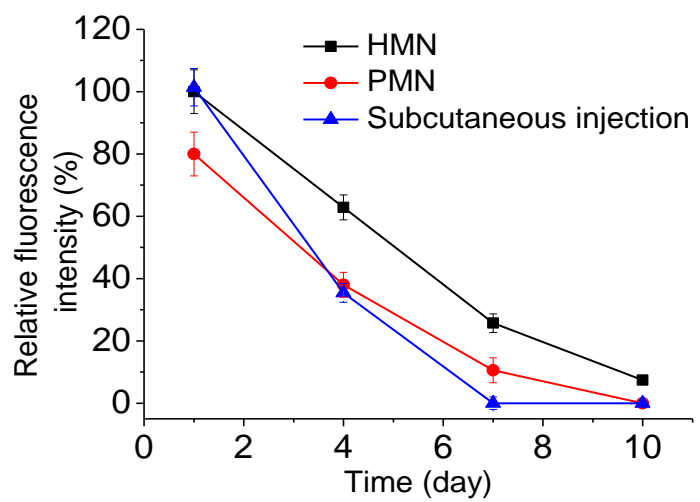

Figure S11. The fluorescence intensity profiles of Dil labeled-exosomes administrated via HMN, PMN, and subcutaneous injection. The fluorescence intensity of Dil labeled-exosomes at Day 1 via the HMN administration was taken as $100 \%$. Data points represent mean $\pm \mathrm{SD}(\mathrm{n}=3)$. Error bars indicate SD. 


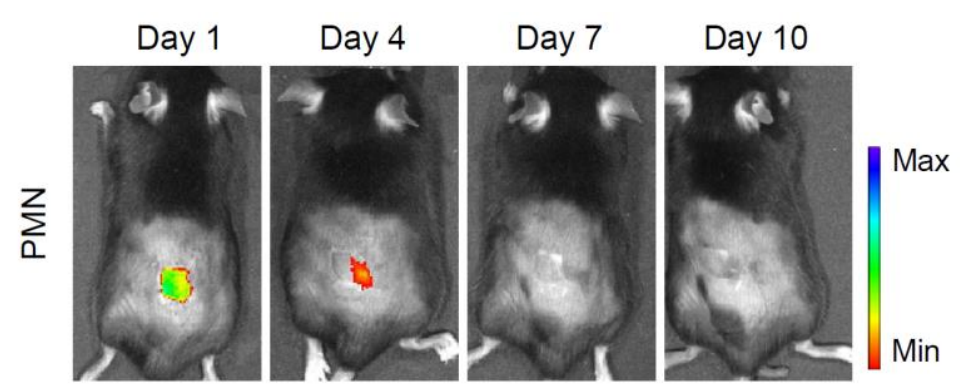

Figure S12. In vivo degradation of PMN after removal of the patch base. The microneedles were fabricated by Cy5.5-labeled keratin.

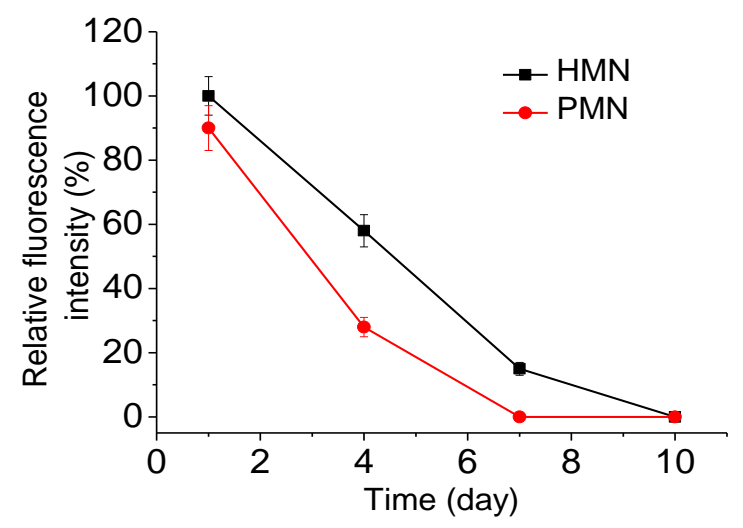

Figure S13. The fluorescence intensity profiles of Cy5.5-labeled microneedles administrated via HMN and PMN. The fluorescence intensity of Cy5.5-labeled microneedles at Day 1 via the HMN administration was taken as $100 \%$. Data points represent mean \pm SD $(n=3)$. Error bars indicate SD.

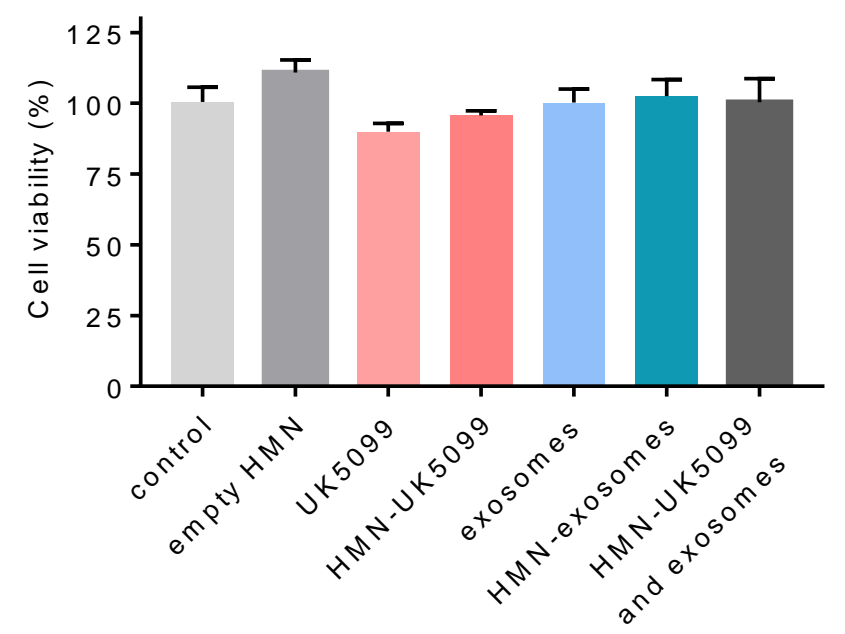

Figure S14. Cell viability assay. Cells were incubated with control (PBS), soak solutions of empty HMN, UK5099-loaded HMN, exosomes-loaded HMN, or UK5099 and exosomes-loaded HMN, respectively. Pure UK5099 and exosomes were used for comparison. Data points represent mean \pm $\mathrm{SD}(\mathrm{n}=4)$. Error bars indicate SD. 


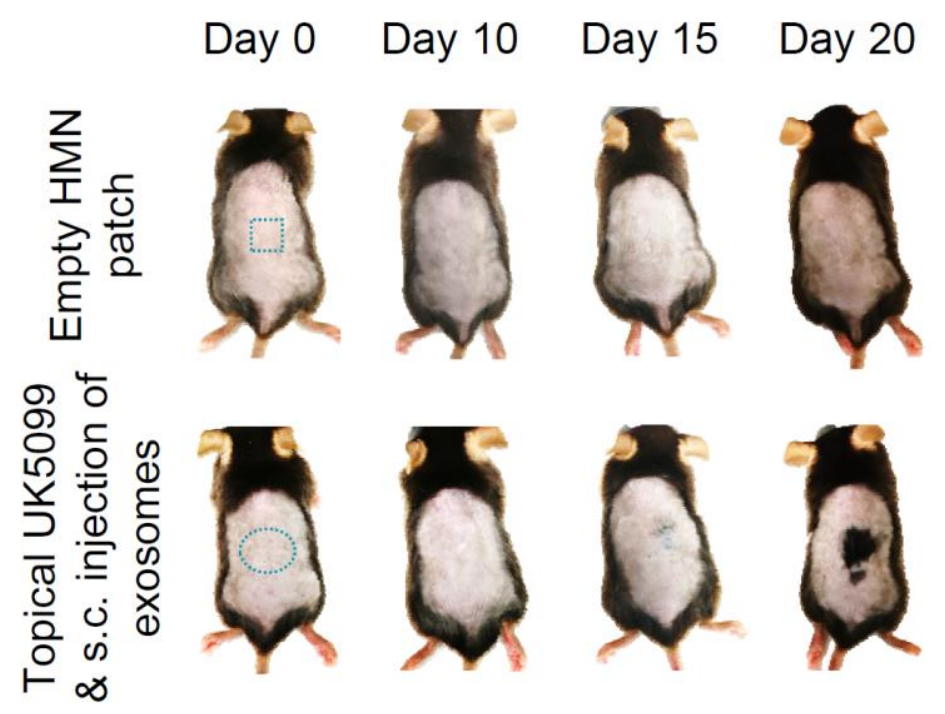

Figure S15. Photographs of mice treated with empty HMN patch, and topical UK5099 combined with subcutaneous injection of exosomes. The treated skin region was marked in blue.

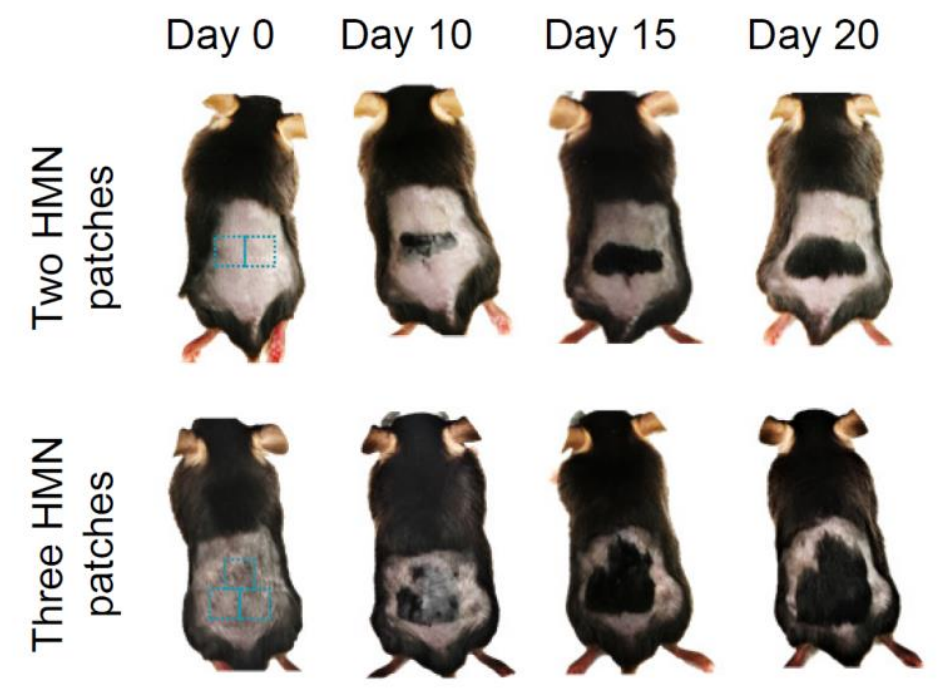

Figure S16. Photographs of mice treated with several HMN patches loaded with UK5099 and exosomes. The treated skin region was marked in blue. 


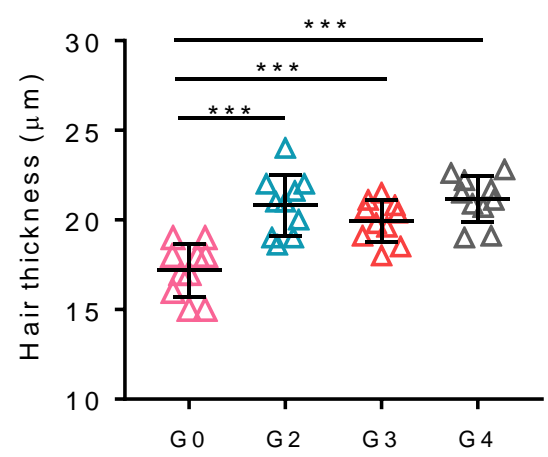

Figure S17. Hair thickness of mice treated by exosomes and UK5099-loaded HMN (G2), UK5099loaded HMN (G3), and exosomes-loaded HMN (G4), by comparison with the wild-type mice (G0). Data points represent mean $\pm \mathrm{SD}(\mathrm{n}=10)$. Error bars indicate SD. $* * * P<0.001$.

\section{G1 G2 G3 G4 G5 G6}

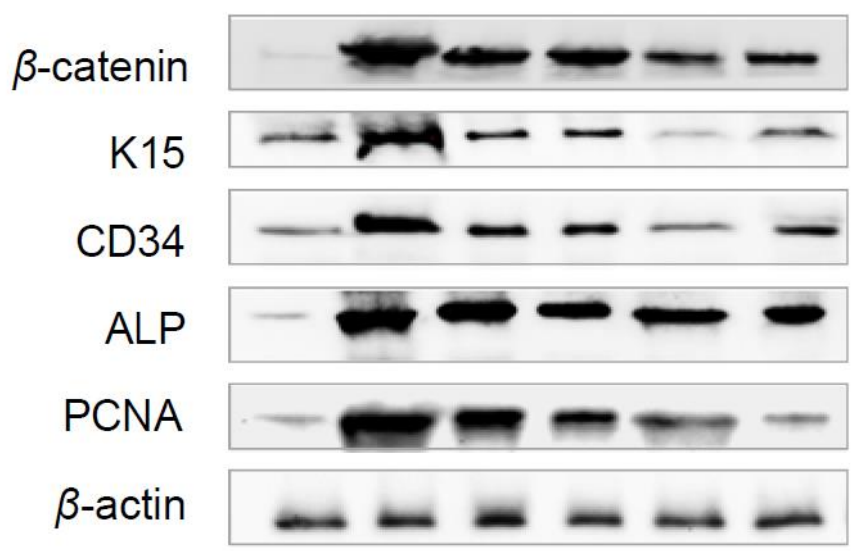

Figure S18. Western blot assay of hair growth-associated protein expression levels in the mice skin treated with exosomes and UK5099-loaded HMN (G2), UK5099-loaded HMN (G3), exosomesloaded HMN (G4), topical UK5099 (G5), subcutaneous injection of exosomes (G6). The mice without any treatment were set as a control (G1). 